\title{
Active Surveillance in Adults with Low-Risk Papillary Thyroid Microcarcinomas: A Prospective Study
}

\author{
Authors \\ Pedro Weslley Rosario, Gabriela Franco Mourão, Maria Regina Calsolari
}

\author{
Affiliation \\ Santa Casa de Belo Horizonte, Minas Gerais, Brazil \\ Key words \\ active surveillance, thyroidectomy, microcarcinoma \\ received $\quad 16.04 .2019$ \\ accepted 12.09.2019 \\ Bibliography \\ DOI https://doi.org/10.1055/a-1015-6684 \\ Horm Metab Res 2019; 51: 703-708 \\ (c) Georg Thieme Verlag KG Stuttgart · New York \\ ISSN 0018-5043 \\ Correspondence \\ P. W. Rosario, MD \\ Ensino e Pesquisa da Santa Casa de Belo Horizonte \\ Rua Domingos Vieira 590 \\ Santa Efigênia CEP 30150-240 \\ Belo Horizonte \\ Minas Gerais \\ Brazil \\ Tel.: + 55/31/32388 819, Fax: + 55/31/32388980 \\ pedrowsrosario@gmail.com
}

\begin{abstract}
The acceptance and results of active surveillance in patients with low-risk papillary thyroid microcarcinomas (PTMC) are unknown in populations other than the Japanese population. This was the objective of the present prospective study. We selected patients $\geq 20$ years who had thyroid nodules $\leq 1.2 \mathrm{~cm}$ with intermediate or high suspicion for malignancy on ultrasonography (US), not located near the recurrent laryngeal nerve and without extrathyroidal invasion or apparent lymph node metastases, whose cytology was suspicious (Bethesda $\mathrm{V}$ ) or diagnostic (Bethesda VI) of papillary thyroid carcinoma. Patients who opted for active surveillance were followed up by biannual US. Fifteen patients ( $18.7 \%$ ) readily opted for surgery and $12(15 \%)$ for active surveillance. Fifty-three patients (66.2\%) delegated the decision or wished to know the doctor's preference before deciding. After the doctor had declared his/ her preference for active surveillance, 50 patients decided to have this management and three to have surgery. Only 1/70 patients exhibited tumor progression (growth associated with a suspicion of extrathyroidal invasion) after 30 months of follow-up. Two patients decided to have surgery during follow-up, although the indication was not defined by the study. $A>50 \%$ reduction in tumor volume was observed in three patients. The study shows that active surveillance can be well accepted if doctors were convinced that it is the best option for patients with low-risk PTMC. At least the short-term results reproduced those observed in other populations, with tumor progression being uncommon.
\end{abstract}

\section{Introduction}

Follow-up instead of immediate fine-needle aspiration (FNA) has been recommended for most adults with thyroid nodules $\leq 1$ without extrathyroidal extension or apparent lymph node involvement on ultrasonography (US) [1-3]. In this situation, active surveillance has been considered when FNA is obtained and is suspicious or diagnostic of non-aggressive papillary thyroid carcinoma (PTC) [1, 3]. Indeed, studies have shown a low frequency of progression of lowrisk papillary thyroid microcarcinomas (PTMC) in adults [3-8]. However, most of these studies involved Japanese and Korean populations, which accounted for more than $80 \%$ of the patients studied [3]. To our knowledge, among studies conducted in other countries, only one North American study [5] and a Columbian study [8] have been published. Population differences in terms of the acceptance of active surveillance instead of immediate intervention and the natural history of low-risk PTMC are possible. In fact, although the American Thyroid Association has endorsed follow-up without FNA of nodules $\leq 1 \mathrm{~cm}$ and the possibility of no immediate intervention in the case of low-risk PTMC, it emphasizes the need to evaluate this management in populations other than the Japanese population [1].

We therefore report here the results of a prospective study that evaluated the acceptance of active surveillance in adult patients diagnosed with low-risk PTMC and the frequency of short-term progression of these non-operated tumors. 


\section{Patients and Methods}

This was a prospective study started in January 2016 whose results were obtained up to 30 June 2019 . The study was approved by the local Research Ethics Committee. The selection criteria and protocol were pre-defined and rigorously followed.

\section{Patients}

Adults (age $\geq 20$ years) with thyroid nodules $\leq 1.2 \mathrm{~cm}$ consecutively seen during the period reported above, who met the inclusion criteria ( $\triangleright$ Fig. 1), were initially selected. An additional evaluation was performed by the author (P.W.R.) to exclude patients classified as high risk and who were not candidates for active surveillance. Six patients were excluded during this step ( $\triangleright$ Fig. 1).

\section{Definition of management}

For analysis of the acceptance of active surveillance, 15 patients who had been referred because they did not wish to undergo surgery (pre-defined option) were excluded. The remaining patients who had not made a decision at the time of inclusion in the study were offered the options of active surveillance or immediate surgery. Two pre-established conditions that needed to be followed were that the doctor would only express his/her preference (which is active surveillance) if requested by the patient and that he/she would not question or argue against the patient's decision for immediate surgery.

\section{Follow-up and tumor progression}

For analysis of tumor progression, patients with a previous preference for follow-up and those who opted for this management on the occasion of the study were included ( $\triangleright$ Fig. 1). US was obtained every 6 months and the following parameters were evaluated: growth $\geq 3 \mathrm{~mm}$, tumor volume increase $\geq 50 \%$ (calculated using the ellipsoid equation: $\pi / 6 \times$ length $\times$ width $\times$ height), suspicion of extrathyroidal invasion [9], and suspicion of lymph node metastases [9-11]. In patients with $\mathrm{TSH}>2.5 \mathrm{mIU} / \mathrm{l}$ in two consecutive measurements, levothyroxine (L-T4) was started to maintain TSH between $0.5-2 \mathrm{mIU} / \mathrm{l}$. This target was the same in the few cases that already used L-T4 $(n=4)$ before the study.

In each patient, the initial US and those obtained during active surveillance were performed by the same examiner. Considering that the tumors had a maximum size of $1.2 \mathrm{~cm}$, increases of $\geq 3 \mathrm{~mm}$ in diameter or $\geq 50 \%$ in volume, as adopted in all previous studies [4-8], would not be attributed to intraobserver variation [12].

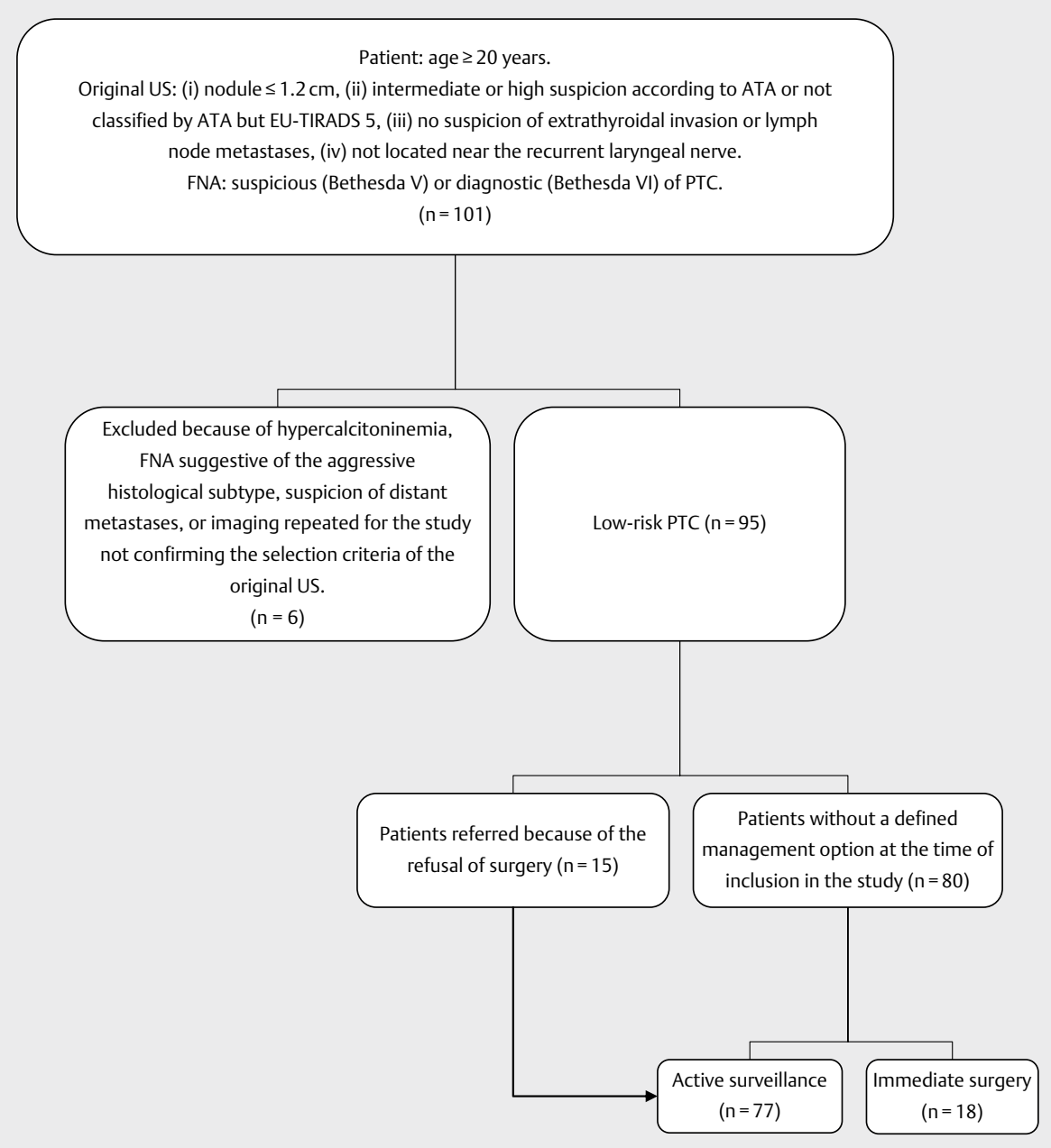

- Fig. 1 Selection of the patients. ATA: American Thyroid Association; EU-TIRADS: European Thyroid Imaging Reporting and Data System; FNA: Fine-needle aspiration; PTC: Papillary thyroid carcinoma; US: Ultrasonography. 


\section{Surgical indication during follow-up}

The following conditions were considered surgical indications: (i) tumor growth associated with a size $>1.2 \mathrm{~cm}$ or suspicion of extrathyroidal invasion, or (ii) appearance of suspicious lymph nodes on US with confirmation of the metastatic nature by FNA. A growth $\geq 3 \mathrm{~mm}$ or tumor volume increase $\geq 50 \%$, but maintaining a size $\leq 1.2 \mathrm{~cm}$ in the absence of a suspicion of extrathyroidal invasion or lymph node metastases, was not considered a surgical indication and the patient remained under active surveillance. Obviously, if the patient expressed the desire for treatment at any time, regardless of the previous criteria, this wish was respected without the doctor arguing against it.

\section{Results}

The characteristics of the patients included in the study are shown in $>$ Table 1.

\section{Acceptance of active surveillance}

In the case of patients who had not made a decision on the occasion of inclusion in the study $(\mathrm{n}=80), 15(18.7 \%)$ readily opted for surgery and 12 (15\%) for active surveillance after the two options were given. Fifty-three patients (66.2\%) delegated the decision to the doctor or wished to know his/her preference before deciding. After the doctor had expressed his/her preference for active surveillance, 50 patients finally opted for this management and three for surgery. Comparing patients who opted for active surveillance versus immediate surgery, a personal history of non-thyroid malignancy and the presence of two tumor foci were significantly more frequent in the latter ( $\triangleright$ Table 2 ). In 8/77 (10.4\%) patients who opted for active surveillance, L-T4 was started to maintain TSH between $0.5-2 \mathrm{mIU} / \mathrm{l}$.

\section{Tumor progression}

During closure of the results, seven patient had not yet undergone the second evaluation (6 months). In addition to the initial assessment, the number of biannual evaluations was 6 in 18 patients, $\geq 5$ in $24, \geq 4$ in $36, \geq 3$ in $45, \geq 2$ in 55, and $\geq 1$ in 70 . Only one patient exhibited tumor progression after 30 months of follow-up, which led to the indication of surgery. In this case, US showed tumor growth associated with a suspicion of extrathyroidal invasion, but no lymph node metastases. Two patients decided to undergo surgery during follow-up, although they had no indication defined by the study. Since there was only one case, we did not analyze pre-

- Table 1 Characteristics of the patients studied.

\begin{tabular}{|c|c|c|}
\hline & $\begin{array}{l}\text { Patients evaluated regarding acceptance of } \\
\text { active surveillance }(n=80)\end{array}$ & $\begin{array}{l}\text { Patients evaluated regarding tumor } \\
\text { progression during active surveillance }(n=77)\end{array}$ \\
\hline \multicolumn{3}{|l|}{ Sex } \\
\hline Female & 65 (81.2\%) & 61 (79.2\%) \\
\hline Male & 15 (18.8\%) & $16(20.8 \%)$ \\
\hline Age [range (median), years] & $25-80(53)$ & $23-81(52)$ \\
\hline Complete higher education & $72(90 \%)$ & $69(89.6 \%)$ \\
\hline History of radiation exposure & $1(1.2 \%)$ & 0 \\
\hline History of non-thyroid malignancy & $16(20 \%)$ & 9 (11.7\%) \\
\hline Family history of PTC & $10(12.5 \%)$ & $8(10.4 \%)$ \\
\hline Associated Hashimoto's thyroiditis & $10(12.5 \%)$ & $10(13 \%)$ \\
\hline Associated Graves' disease & 0 & 0 \\
\hline Multinodular disease & $15(18.7 \%)$ & $14(18.2 \%)$ \\
\hline \multicolumn{3}{|l|}{ FNA } \\
\hline Bethesda V & $16(20 \%)$ & $16(20.7 \%)$ \\
\hline Bethesda VI & $64(80 \%)$ & $61(79.2 \%)$ \\
\hline \multicolumn{3}{|l|}{ Tumor size } \\
\hline$\leq 1 \mathrm{~cm}$ & $72(90 \%)$ & $69(89.6 \%)$ \\
\hline $1-1.2 \mathrm{~cm}$ & $8(10 \%)$ & $8(10.4 \%)$ \\
\hline \multicolumn{3}{|l|}{ Number of known tumor foci } \\
\hline 1 & $75(93.7 \%)$ & $76(98.7 \%)$ \\
\hline 2 & $5(6.2 \%)$ & $1(1.3 \%)$ \\
\hline \multicolumn{3}{|c|}{ Appropriateness for active surveillance (24) } \\
\hline Ideal & $5(6.2 \%)$ & $5(6.5 \%)$ \\
\hline Appropriate & $75(93.7 \%)$ & $72(93.5 \%)$ \\
\hline
\end{tabular}

FNA: Fine-needle aspiration; PTC: Papillary thyroid carcinoma. 
- Table 2 Comparison of patients who opted for active surveillance versus immediate surgery.

\begin{tabular}{|c|c|c|c|}
\hline & \multirow[t]{2}{*}{ Immediate surgery $(n=18)$} & \multicolumn{2}{|c|}{ Active surveillance } \\
\hline & & Decision during the study $(n=62)$ & All $(n=77)$ \\
\hline \multicolumn{4}{|l|}{ Sex } \\
\hline Female & $15(83.3 \%)$ & $50(80.6 \%)$ & $61(79.2 \%)$ \\
\hline Male & $3(16.7 \%)$ & $12(19.3 \%)$ & $16(20.8 \%)$ \\
\hline Age [range (median), years] & $25-78(51)$ & $26-80(52)$ & $23-81(52)$ \\
\hline Complete higher education & $16(88.9 \%)$ & $56(90.3 \%)$ & $69(89.6 \%)$ \\
\hline History of radiation exposure & $1(5.5 \%)$ & 0 & 0 \\
\hline History of non-thyroid malignancy & $8(44.4 \%)$ & $8(12.9 \%)^{a}$ & $9(11.7 \%)^{b}$ \\
\hline Family history of PTC & $4(22.2 \%)$ & $6(9.7 \%)$ & $8(10.4 \%)$ \\
\hline Associated Hashimoto's thyroiditis & $2(11.1 \%)$ & $8(12.9 \%)$ & $10(13 \%)$ \\
\hline Associated Graves’s disease & 0 & 0 & 0 \\
\hline Multinodular disease & $4(22.2 \%)$ & $11(17.7 \%)$ & $14(18.2 \%)$ \\
\hline \multicolumn{4}{|l|}{ FNA } \\
\hline Bethesda V & $4(22.2 \%)$ & $12(19.3 \%)$ & $16(20.8 \%)$ \\
\hline Bethesda VI & $14(77.8 \%)$ & $50(80.6 \%)$ & $61(79.2 \%)$ \\
\hline \multicolumn{4}{|l|}{ Tumor size } \\
\hline$\leq 1 \mathrm{~cm}$ & $16(88.9 \%)$ & $56(90.3 \%)$ & $69(89.6 \%)$ \\
\hline $1-1.2 \mathrm{~cm}$ & $2(11.1 \%)$ & $6(9.7 \%)$ & $8(10.4 \%)$ \\
\hline \multicolumn{4}{|l|}{ Number of known tumor foci } \\
\hline 1 & $14(77.8 \%)$ & $61(98.4 \%)$ & $76(98.7 \%)$ \\
\hline 2 & $4(22.2 \%)$ & $1(1.6 \%)^{c}$ & $1(1.3 \%)^{d}$ \\
\hline \multicolumn{4}{|c|}{ Appropriateness for active surveillance (24) } \\
\hline Ideal & $1(5.5 \%)$ & $4(6.4 \%)$ & $5(6.5 \%)$ \\
\hline Appropriate & $17(94.4 \%)$ & $58(93.5 \%)$ & $72(93.5 \%)$ \\
\hline
\end{tabular}

dictors of tumor progression. Interestingly, three patients had a $>50 \%$ reduction in tumor volume.

\section{Additional information}

Among adults $\geq 20$ years with nodules $\leq 1.2 \mathrm{~cm}$ who were submitted to FNA but excluded from the study because cytology was not suspicious or diagnostic of PTC, 21 patients had highly suspicious nodules on US [1] and Bethesda cytology I (on two occasions), III (on two occasions), or IV. In these situations (combination of US and FNA), the risk of malignancy exceeds $50 \%$ in our experience [13-16], and the patients were also followed up by US every 6 months. The number of biannual evaluations (after initial assessment) was 6 in 5 patients, $\geq 5$ in $7, \geq 4$ in $10, \geq 3$ in $12, \geq 2$ in 15 , and $\geq 1$ in 21 . None of the patients exhibited progression of the nodule or dropped out of active surveillance.

Although presenting criteria for active surveillance, 20 patients opted for surgery (18 at the beginning of the study and 2 during follow-up). According to the 8th edition of the TNM classification [17], none of the tumors was staged as T3b or T4 and all tumors were $\leq 1 \mathrm{~cm}$ on histology (T1a). Central lymph node involvement was suspected during perioperative evaluation and histology con- firmed metastases in 1 patient ( $5 \%$; cN1a). None of the patients had elevated serum thyroglobulin or a suspicion of metastases in the first assessment and 12 months after surgery. Distant metastases were therefore considered to be absent (M0).

\section{Discussion}

Active surveillance was well accepted (80\%) in the present study. FNA demonstrating malignancy was necessary for inclusion in the study. It is possible that more patients would opt for follow-up if they had only a diagnosis of a small thyroid nodule for which the doctor did not request a biopsy instead of receiving a diagnosis of malignancy after being submitted to FNA [18]. Thus, one strategy that could increase the acceptance of active surveillance is a follow-up in which FNA is not obtained for suspicious nodules $\leq 1 \mathrm{~cm}$ without extrathyroidal extension or apparent lymph node involvement on US and that do not affect a worrisome site. In fact, this is the current recommendation of ATA [1], ETA [2], and some experts [3]. It is also important to stress that, because this was a prospective study using a standardized approach, the doctor did not argue in favor of active surveillance when it was not requested or when 
the patient decided to have surgery. In clinical practice in which the doctor could argue in favor of his/her preference (in this case, active surveillance), more patients might be convinced to opt for follow-up.

With respect to factors that may have contributed to the acceptance of active surveillance, although it is medical-scientific literature, the various guidelines on the subject are accessible on the internet and there has been absolute consensus in recent years that FNA is not necessary in the case of nodules $\leq 1 \mathrm{~cm}$ and that surgery is not readily required in low-risk PTMC. We believe that this conveys to the patient the idea that active surveillance is already a reality and a consistent option rather than an exceptional or still experimental management. There is also a growing body of literature showing that some patients may remain symptomatic or unsatisfied with L-T4 replacement as it is currently done. We believe that this causes greater resistance in patients to remove, even if partial, their functionally normal gland. We highlight these two aspects because, although not objectively and quantitatively evaluated, many patients reported to have searched the internet for information on these topics before making their decision. However, we recognize that the importance of these factors is probably influenced directly by the educational level of the patients and these factors had therefore greater relevance in this study.

Another factor that also seems to be important is convincing the doctor. Most of the patients (2/3) wanted to know the doctor's preference before making their decision and, after the doctor expressed his/her preference, almost all patients followed the same option or trusted the doctor with the best choice. According to the study protocol, in these cases, the doctor did not omit his/her opinion and clearly expressed the preference for active surveillance. Even if presenting the two options to the patients, we believe that the acceptance of active surveillance is much compromised if the physician does not transmit safety regarding this option, and especially if he/she expresses preference, even if hidden, for surgery. The experience of the Kuma Hospital in Japan shows that the frequency of active surveillance in PTMC increased from $30 \%$ between 1993-1997 to $88 \%$ between 2014-2016 [19]. Interestingly, in the last triennium, the frequency of active surveillance reached $97 \%$ among endocrinologists who prefer it more often than surgeons [19]. This observation and the results of the present study suggest that acceptance by the patient does not seem to be a problem when it is addressed by a physician who is convinced that active surveillance is the best option.

Regarding tumor progression, we recognize the short study period. Nevertheless, the initial results are similar to those reported in series with a longer follow-up time involving other populations [4-7]. A recent review of previous studies showed the absence of distant metastases in approximately 2500 patients with low-risk PTMC submitted to active surveillance, and only $1 \%$ of the patients developed lymph node metastases apparent on US [3]. The frequency of tumor growth (increase $\geq 3 \mathrm{~mm}$ ) was on average $5 \%$ [3], even in studies with longer follow-up $[4,6]$. Since we had only one case of tumor progression, it was not possible to analyze predictive factors. As the most commonly found predictor, patient age has been related to the risk of tumor growth, which is higher in young individuals [4-7]. Interestingly, as observed in three of our patients, tumor reduction has also been reported in other series at a frequency ranging from $5-15 \%[5,6,20,21]$.
A reassuring fact in active surveillance is that disease-free survival in patients subsequently undergoing surgery does not seem to be compromised by postponement of the procedure. Taken together the data of 4 series [4-7], the review cited above showed that among 303 patients who underwent surgery after a period of active surveillance, highlighting that most of them did not receive radioactive iodine, only one case of short-term local recurrence (0.35\%) was observed [3].

Finally, we included tumors measuring up to $1.2 \mathrm{~cm}$ for active surveillance. Other series also included tumors of up to $1.5 \mathrm{~cm}$ $[5,8,22]$ and no difference in outcomes has been found so far for microcarcinomas and PTC between 1 and $1.5 \mathrm{~cm}$ [5, 22]. In agreement, in the case of hypoechoic nodules (intermediate suspicion), FNA was traditionally recommended for nodules $>1 \mathrm{~cm}$, while the new recommendations of the American College of Radiology now indicate FNA for nodules $>1.5 \mathrm{~cm}$ [23]. It should also be noted that only 6/95 patients (6.3\%) would be considered "ideal candidates" [24] for active surveillance (data not shown). In the study of Tuttle et al. [5], only $5 \%$ of the patients were "ideal candidates". This fact shows that, in practice, the adoption of this restricted criterion may impede active surveillance.

The results suggest that active surveillance can be very well accepted if doctors are convinced that it is the best option for patients with low-risk PTMC. Regarding the progression of these tumors, at least in the short term, the results were similar to those observed in other populations, with tumor progression being uncommon.

\section{Funding}

This work was supported by the National Council for Scientific and Technological Development (CNPq).

\section{Conflict of Interest}

The authors declare that they have no conflict of interest.

\section{References}

[1] Haugen BR, Alexander EK, Bible KC et al. 2015 American Thyroid Association management guidelines for adult patients with thyroid nodules and differentiated thyroid cancer: The American Thyroid Association guidelines task force on thyroid nodules and differentiated thyroid cancer. Thyroid 2016; 26: 1-133

[2] Russ G, Bonnema S], Erdogan MF et al. European Thyroid Association guidelines for ultrasound malignancy risk stratification of thyroid nodules in adults: The EU-TIRADS. Eur Thyroid J 2017; 6: 225-237

[3] Rosario PW, Ward LS, Graf $\mathrm{H}$ et al. Thyroid nodules $\leq 1 \mathrm{~cm}$ and papillary thyroid microcarcinomas: Brazilian experts opinions. Arch Endocrinol Metab 2019; Sep 2. pii S2359-39972019005007102 doi:10.20945/2359-3997000000166 [Epub ahead of print]

[4] Ito Y, Miyauchi A, Kihara M et al. Patient age is significantly related to the progression of papillary microcarcinoma of the thyroid under observation. Thyroid 2014; 24: 27-34

[5] Tuttle RM, Fagin JA, Minkowitz G et al. Natural history and tumor volume kinetics of papillary thyroid cancers during active surveillance. JAMA Otolaryngol Head Neck Surg 2017; 143: 1015-1020 
[6] Sugitani I. Active surveillance for very low-risk papillary thyroid carcinoma: Experience and perspectives from Japan. Ann Thyroid 2018; 3: 26

[7] Sanabria A. Active Surveillance in Thyroid microcarcinoma in a latin-american cohort. JAMA Otolaryngol Head Neck Surg 2018; 144: 947-948

[8] Oh HS, Ha J, Kim HI et al. Active surveillance of low-risk papillary thyroid microcarcinoma: A multi-center cohort study in Korea. Thyroid 2018; 28: 1587-1594

[9] Rosario PWS, Mourão GF, Oliveira PHL et al. Are papillary thyroid carcinomas that are candidates for active surveillance in fact classical microcarcinomas restricted to the gland? Eur. Thyroid J 2018; 7: 258-261

[10] Rosario PW, de Faria S, Bicalho L et al. Ultrasonographic differentiation between metastatic and benign lymph nodes in patients with papillary thyroid carcinoma. J Ultrasound Med 2005; 24: 1385-1389

[11] Rosario PW, Tavares WC, Borges MA et al. Ultrasonographic differentiation of cervical lymph nodes in patients with papillary thyroid carcinoma after thyroidectomy and radioiodine ablation: A prospective study. Endocr Pract 2014; 20: 293-298

[12] Lee HJ, Yoon DY, Seo YL et al. Intraobserver and interobserver variability in ultrasound measurements of thyroid nodules. J Ultrasound Med 2018; 37: 173-178

[13] Rosario PW, Salles DS, Bessa B et al. Contribution of scintigraphy and ultrasonography to the prediction of malignancy in thyroid nodules with indeterminate cytology. Arq Bras Endocrinol Metabol 2010; 54: 56-59

[14] Rosario PW. Thyroid nodules with atypia or follicular lesions of undetermined significance (Bethesda Category III): Importance of ultrasonography and cytological subcategory. Thyroid 2014; 24: 1115-1120

[15] Rosario PW, Penna GC, Calsolari MR. Predictive factors of malignancy in thyroid nodules with repeatedly nondiagnostic cytology (Bethesda category I): Value of ultrasonography. Horm Metab Res 2014; 46: 294-298
[16] Rocha TG, Rosario PW, Silva AL et al. Ultrasonography classification of the american thyroid association for predicting malignancy in thyroid nodules $>1 \mathrm{~cm}$ with indeterminate cytology: A prospective study. Horm Metab Res 2018; 50: 597-601

[17] Tuttle M, Morris LF, Haugen B et al. AJCC Cancer Staging Manual. In: Amin MB, Edge SB, Greene FL eds 8th ed. New York, NY: Springer; 2017: 1-19

[18] Dixon PR, Tomlinson G, Pasternak JD et al. The role of disease label in patient perceptions and treatment decisions in the setting of low-risk malignant neoplasms. JAMA Oncol 2019; 5: 817-823

[19] Ito Y, Miyauchi A, Kudo T et al. Trends in the implementation of active surveillance for low-risk papillary thyroid microcarcinomas at Kuma Hospital: Gradual increase and heterogeneity in the acceptance of this new management option. Thyroid 2018; 28: 488-495

[20] Ito Y, Uruno T, Nakano K, Takamura Y et al. An observation trial without surgical treatment in patients with papillary microcarcinoma of the thyroid. Thyroid 2003; 13: 381-387

[21] Kwon H, Oh HS, Kim M et al. Active surveillance for patients with papillary thyroid microcarcinoma: A single center's experience in Korea. J Clin Endocrinol Metab 2017; 102: 1917-1925

[22] Sakai T, Sugitani I, Ebina A et al. Active surveillance for T1bN0M0 papillary thyroid carcinoma. Thyroid 2019; 29: 59-63

[23] Tessler FN, Middleton WD, Grant EG et al. ACR Thyroid Imaging, Reporting and Data System (TI-RADS): White Paper of the ACR TI-RADS Committee. J Am Coll Radiol. 2017: 14: 587-595

[24] Brito JP, Ito Y, Miyauchi A et al. A clinical framework to facilitate risk stratification when considering an active surveillance alternative to immediate biopsy and surgery in papillary microcarcinoma. Thyroid 2016; $26: 144-149$ 